\title{
Again on the possible compositeness of the photon.*
}

\author{
Valeri V. Dvoeglazov \\ Escuela de Física, Universidad Autónoma de Zacatecas \\ Apartado Postal C-580, Zacatecas 98068 Zac., México \\ E-mail:valeri@ahobon.reduaz.mx
}

URL: http://ahobon.reduaz.mx/ valeri/valeri.htm

(February, 1998)

\begin{abstract}
We construct the transversal and longitudinal 4-vector potentials and strengths $F^{\mu \nu}$ in the momentum representation on using the second-type 4spinors recently proposed by Ahluwalia. Questions of relevance of this kind of theories to the correct photon statistics are briefly discussed.
\end{abstract}

\section{INTRODUCTION}

In the papers [1] 6] the hypothesis was discussed that a photon is a composite state of a pair of neutral particles of the $(1 / 2,0) \oplus(0,1 / 2)$ representation. Unfortunately, the matters related to the correct photon statistics have not been clarified in the papers on "the neutrino theory of light". Recently, after the study of previous papers [7,8] I asked several questions [9] which might be relevant to this kind of field models. In the present note I give a formal basis to some of my previous thoughts. Main objections were: 1) why did one use the charged particle (electron-positron) 4-spinors and the Dirac current only in order to construct photon states? 2) why were physicists so unpleasant to accept that the electromagentic 4-vector may be longitudinal? 3) why did the physicists almost not explore the possibility of using the electric and magnetic fields $\mathbf{E}$ and $\mathbf{B}$ in order to describe the light in the quantum field theory?

\footnotetext{
*Submitted to "International Journal Theoretical Physics"
} 


\section{CLASSICAL FIELDS FOR A COMPOSITE PHOTON-NOTOPH}

Here, we first construct the composite photons on using the 2-nd kind spinors [10,11]. On the basis of the formulas given in the Appendices A and B one obtains

$$
\begin{aligned}
& \bar{\lambda}_{\uparrow}^{S}\left(p^{\mu}\right) \gamma^{\mu} \lambda_{\uparrow}^{S}\left(p^{\mu}\right)=\bar{\lambda}_{\uparrow}^{A}\left(p^{\mu}\right) \gamma^{\mu} \lambda_{\uparrow}^{A}\left(p^{\mu}\right)=\frac{m}{N}\left[u^{\mu}\left(\mathbf{p}, 0_{t}\right)-u^{\mu}(\mathbf{p}, 0)\right], \\
& \bar{\lambda}_{\downarrow}^{S}\left(p^{\mu}\right) \gamma^{\mu} \lambda_{\downarrow}^{S}\left(p^{\mu}\right)=\bar{\lambda}_{\downarrow}^{A}\left(p^{\mu}\right) \gamma^{\mu} \lambda_{\downarrow}^{A}\left(p^{\mu}\right)=\frac{m}{N}\left[u^{\mu}\left(\mathbf{p}, 0_{t}\right)+u^{\mu}(\mathbf{p}, 0)\right], \\
& \bar{\lambda}_{\uparrow}^{S}\left(p^{\mu}\right) \gamma^{\mu} \lambda_{\downarrow}^{S}\left(p^{\mu}\right)=\bar{\lambda}_{\downarrow}^{S}\left(p^{\mu}\right) \gamma^{\mu} \lambda_{\uparrow}^{S}\left(p^{\mu}\right)=\frac{m}{N \sqrt{2}}\left[u^{\mu}(\mathbf{p},+1)-u^{\mu}(\mathbf{p},-1)\right], \\
& \bar{\lambda}_{\uparrow}^{S}\left(p^{\mu}\right) \gamma^{\mu} \lambda_{\downarrow}^{A}\left(p^{\mu}\right)=-\bar{\lambda}_{\downarrow}^{S}\left(p^{\mu}\right) \gamma^{\mu} \lambda_{\uparrow}^{A}\left(p^{\mu}\right)=-\frac{m}{N \sqrt{2}}\left[u^{\mu}(\mathbf{p},+1)+u^{\mu}(\mathbf{p},-1)\right] .
\end{aligned}
$$

After simple alebraic transformations one can write the $(1 / 2,1 / 2)$ momentum-space function, for instance, in the following forms $\left(a_{1}+a_{2}=1, a_{3}+a_{4}=1\right):^{1}$

$$
\begin{aligned}
& u^{\mu}(\mathbf{p},+1)=\frac{N}{m \sqrt{2}}\left\{a_{1}\left[\bar{\lambda}_{\uparrow}^{S}\left(p^{\mu}\right) \gamma^{\mu}\left(1+\gamma^{5}\right) \lambda_{\downarrow}^{S}\left(p^{\mu}\right)\right]+a_{2}\left[\bar{\lambda}_{\downarrow}^{S}\left(p^{\mu}\right) \gamma^{\mu}\left(1-\gamma^{5}\right) \lambda_{\uparrow}^{S}\left(p^{\mu}\right)\right]\right\}= \\
&=\frac{N}{m \sqrt{2}}\left\{a_{1}\left[\bar{\lambda}_{\uparrow}^{A}\left(p^{\mu}\right) \gamma^{\mu}\left(1+\gamma^{5}\right) \lambda_{\downarrow}^{A}\left(p^{\mu}\right)\right]+a_{2}\left[\bar{\lambda}_{\downarrow}^{A}\left(p^{\mu}\right) \gamma^{\mu}\left(1-\gamma^{5}\right) \lambda_{\uparrow}^{A}\left(p^{\mu}\right)\right]\right\}, \\
& u^{\mu}(\mathbf{p},-1)=-\frac{N}{m \sqrt{2}}\left\{a_{3}\left[\bar{\lambda}_{\uparrow}^{S}\left(p^{\mu}\right) \gamma^{\mu}\left(1-\gamma^{5}\right) \lambda_{\downarrow}^{S}\left(p^{\mu}\right)\right]+a_{4}\left[\bar{\lambda}_{\downarrow}^{S}\left(p^{\mu}\right) \gamma^{\mu}\left(1+\gamma^{5}\right) \lambda_{\uparrow}^{S}\left(p^{\mu}\right)\right]\right\}= \\
&=-\frac{N}{m \sqrt{2}}\left\{a_{3}\left[\bar{\lambda}_{\uparrow}^{A}\left(p^{\mu}\right) \gamma^{\mu}\left(1-\gamma^{5}\right) \lambda_{\downarrow}^{A}\left(p^{\mu}\right)\right]+a_{4}\left[\bar{\lambda}_{\downarrow}^{A}\left(p^{\mu}\right) \gamma^{\mu}\left(1+\gamma^{5}\right) \lambda_{\uparrow}^{A}\left(p^{\mu}\right)\right]\right\}, \\
& u^{\mu}(\mathbf{p}, 0)= \frac{N}{2 m}\left[\bar{\lambda}_{\downarrow}^{S}\left(p^{\mu}\right) \gamma^{\mu} \lambda_{\downarrow}^{S}\left(p^{\mu}\right)-\bar{\lambda}_{\uparrow}^{S}\left(p^{\mu}\right) \gamma^{\mu} \lambda_{\uparrow}^{S}\left(p^{\mu}\right)\right]= \\
&=\frac{N}{2 m}\left[\bar{\lambda}_{\downarrow}^{A}\left(p^{\mu}\right) \gamma^{\mu} \lambda_{\downarrow}^{A}\left(p^{\mu}\right)-\bar{\lambda}_{\uparrow}^{A}\left(p^{\mu}\right) \gamma^{\mu} \lambda_{\uparrow}^{A}\left(p^{\mu}\right)\right], \\
& u^{\mu}\left(\mathbf{p}, 0_{t}\right)= \\
&=\frac{N}{2 m}\left[\bar{\lambda}_{\uparrow}^{S}\left(p^{\mu}\right) \gamma^{\mu} \lambda_{\uparrow}^{S}\left(p^{\mu}\right)+\bar{\lambda}_{\downarrow}^{S}\left(p^{\mu}\right) \gamma^{\mu} \lambda_{\downarrow}^{S}\left(p^{\mu}\right)\right]= \\
&=\frac{N}{2 m}\left[\bar{\lambda}_{\uparrow}^{A}\left(p^{\mu}\right) \gamma^{\mu} \lambda_{\uparrow}^{A}\left(p^{\mu}\right)+\bar{\lambda}_{\downarrow}^{A}\left(p^{\mu}\right) \gamma^{\mu} \lambda_{\downarrow}^{A}\left(p^{\mu}\right)\right] .
\end{aligned}
$$

Tensor currents may also be expressed by means of the electric/magnetic strengths:

$$
\begin{aligned}
& \bar{\lambda}_{\uparrow}^{S}\left(p^{\mu}\right) \sigma^{\mu \nu} \lambda_{\downarrow}^{S}\left(p^{\mu}\right)=\bar{\lambda}_{\downarrow}^{S}\left(p^{\mu}\right) \sigma^{\mu \nu} \lambda_{\uparrow}^{S}\left(p^{\mu}\right)=-\frac{2 i m}{N}\left(\begin{array}{c}
\mathbf{E}^{(+)}(\mathbf{p}, 0) \\
\mathbf{B}^{(+)}(\mathbf{p}, 0)
\end{array}\right), \\
& \bar{\lambda}_{\uparrow}^{S}\left(p^{\mu}\right) \sigma^{\mu \nu} \lambda_{\downarrow}^{A}\left(p^{\mu}\right)=\bar{\lambda}_{\downarrow}^{S}\left(p^{\mu}\right) \sigma^{\mu \nu} \lambda_{\uparrow}^{A}\left(p^{\mu}\right)=\frac{2 m}{N}\left(\begin{array}{c}
-\mathbf{B}^{(+)}(\mathbf{p}, 0) \\
\mathbf{E}^{(+)}(\mathbf{p}, 0)
\end{array}\right)=
\end{aligned}
$$

\footnotetext{
${ }^{1}$ For the sake of the general consideration it is assumed the neutrino states to be massive.
} 


$$
\begin{gathered}
=i \Gamma^{0} \Gamma^{5} \bar{\lambda}_{\uparrow}^{S}\left(p^{\mu}\right) \sigma^{\mu \nu} \lambda_{\downarrow}^{S}\left(p^{\mu}\right)=i \Gamma^{0} \Gamma^{5} \bar{\lambda}_{\downarrow}^{S}\left(p^{\mu}\right) \sigma^{\mu \nu} \lambda_{\uparrow}^{S}\left(p^{\mu}\right), \\
\bar{\lambda}_{\uparrow}^{S}\left(p^{\mu}\right) \sigma^{\mu \nu} \lambda_{\uparrow}^{S}\left(p^{\mu}\right)=-\frac{\sqrt{2} i m}{N}\left(\begin{array}{l}
{\left[\mathbf{E}^{(+)}(\mathbf{p},+1)-\mathbf{E}^{(+)}(\mathbf{p},-1)\right]-i\left[\mathbf{B}^{(+)}(\mathbf{p},+1)+\mathbf{B}^{(+)}(\mathbf{p},-1)\right]} \\
i\left[\mathbf{E}^{(+)}(\mathbf{p},+1)+\mathbf{E}^{(+)}(\mathbf{p},-1)\right]+\left[\mathbf{B}^{(+)}(\mathbf{p},+1)-\mathbf{B}^{(+)}(\mathbf{p},-1)\right]
\end{array}\right) \\
\bar{\lambda}_{\uparrow}^{S}\left(p^{\mu}\right) \sigma^{\mu \nu} \lambda_{\uparrow}^{A}\left(p^{\mu}\right)=i \Gamma^{0} \Gamma^{5} \bar{\lambda}_{\uparrow}^{S}\left(p^{\mu}\right) \sigma^{\mu \nu} \lambda_{\uparrow}^{S}\left(p^{\mu}\right), \\
\bar{\lambda}_{\downarrow}^{S}\left(p^{\mu}\right) \sigma^{\mu \nu} \lambda_{\downarrow}^{S}\left(p^{\mu}\right)=\frac{\sqrt{2} i m}{N}\left(\begin{array}{l}
{\left[\mathbf{E}^{(+)}(\mathbf{p},+1)-\mathbf{E}^{(+)}(\mathbf{p},-1)\right]+i\left[\mathbf{B}^{(+)}(\mathbf{p},+1)+\mathbf{B}^{(+)}(\mathbf{p},-1)\right]} \\
-i\left[\mathbf{E}^{(+)}(\mathbf{p},+1)+\mathbf{E}^{(+)}(\mathbf{p},-1)\right]+\left[\mathbf{B}^{(+)}(\mathbf{p},+1)-\mathbf{B}^{(+)}(\mathbf{p},-1)\right]
\end{array}\right) \\
\bar{\lambda}_{\downarrow}^{S}\left(p^{\mu}\right) \sigma^{\mu \nu} \lambda_{\downarrow}^{A}\left(p^{\mu}\right)=i \Gamma^{0} \Gamma^{5} \bar{\lambda}_{\downarrow}^{S}\left(p^{\mu}\right) \sigma^{\mu \nu} \lambda_{\downarrow}^{S}\left(p^{\mu}\right) .
\end{gathered}
$$

In the above formulas and below one has

$$
\Gamma^{5}=\left(\begin{array}{cc}
\mathbb{1}_{3 \times 3} & 0 \\
0 & -\mathbb{1}_{3 \times 3}
\end{array}\right), \quad \Gamma^{0}=\left(\begin{array}{cc}
0 & \mathbb{1}_{3 \times 3} \\
\mathbb{1}_{3 \times 3} & 0
\end{array}\right)
$$

. Hence, we have

$$
\begin{aligned}
\mathbf{E}^{i(+)}(\mathbf{p}, 0) & =\frac{i N}{2 m}\left[c_{1} \bar{\lambda}_{\uparrow}^{S}\left(p^{\mu}\right) \sigma^{0 i} \lambda_{\downarrow}^{S}\left(p^{\mu}\right)+c_{2} \bar{\lambda}_{\downarrow}^{S}\left(p^{\mu}\right) \sigma^{0 i} \lambda_{\uparrow}^{S}\left(p^{\mu}\right)\right]+ \\
& +\frac{N}{4 m} \epsilon^{i j k}\left[c_{3} \bar{\lambda}_{\uparrow}^{S}\left(p^{\mu}\right) \sigma^{j k} \lambda_{\downarrow}^{A}\left(p^{\mu}\right)+c_{4} \bar{\lambda}_{\downarrow}^{S}\left(p^{\mu}\right) \sigma^{j k} \lambda_{\uparrow}^{A}\left(p^{\mu}\right)\right], \\
\mathbf{E}^{(+)}(\mathbf{p},+1) & =c_{5}\left\{\frac{i N}{4 \sqrt{2} m}\left[\bar{\lambda}_{\uparrow}^{S}\left(p^{\mu}\right) \sigma^{0 i} \lambda_{\uparrow}^{S}\left(p^{\mu}\right)-\bar{\lambda}_{\downarrow}^{S}\left(p^{\mu}\right) \sigma^{0 i} \lambda_{\downarrow}^{S}\left(p^{\mu}\right)\right]+\right. \\
& \left.+\frac{N}{8 \sqrt{2} m} \epsilon^{i j k}\left[\bar{\lambda}_{\uparrow}^{S}\left(p^{\mu}\right) \sigma^{j k} \lambda_{\uparrow}^{S}\left(p^{\mu}\right)+\bar{\lambda}_{\downarrow}^{S}\left(p^{\mu}\right) \sigma^{j k} \lambda_{\downarrow}^{S}\left(p^{\mu}\right)\right]\right\}+ \\
& +c_{6}\left\{\frac{i N}{4 \sqrt{2} m}\left[\bar{\lambda}_{\uparrow}^{S}\left(p^{\mu}\right) \sigma^{0 i} \lambda_{\uparrow}^{A}\left(p^{\mu}\right)+\bar{\lambda}_{\downarrow}^{S}\left(p^{\mu}\right) \sigma^{0 i} \lambda_{\downarrow}^{A}\left(p^{\mu}\right)\right]+\right. \\
& \left.+\frac{N}{8 \sqrt{2} m} \epsilon^{i j k}\left[\bar{\lambda}_{\uparrow}^{S}\left(p^{\mu}\right) \sigma^{j k} \lambda_{\uparrow}^{A}\left(p^{\mu}\right)-\bar{\lambda}_{\downarrow}^{S}\left(p^{\mu}\right) \sigma^{j k} \lambda_{\downarrow}^{A}\left(p^{\mu}\right)\right]\right\}, \\
\mathbf{E}^{(+)}(\mathbf{p},-1) & =c_{5}\left\{\frac{-i N}{4 \sqrt{2} m}\left[\bar{\lambda}_{\uparrow}^{S}\left(p^{\mu}\right) \sigma^{0 i} \lambda_{\uparrow}^{S}\left(p^{\mu}\right)-\bar{\lambda}_{\downarrow}^{S}\left(p^{\mu}\right) \sigma^{0 i} \lambda_{\downarrow}^{S}\left(p^{\mu}\right)\right]+\right. \\
& \left.+\frac{N}{8 \sqrt{2} m} \epsilon^{i j k}\left[\bar{\lambda}_{\uparrow}^{S}\left(p^{\mu}\right) \sigma^{j k} \lambda_{\uparrow}^{S}\left(p^{\mu}\right)+\bar{\lambda}_{\downarrow}^{S}\left(p^{\mu}\right) \sigma^{j k} \lambda_{\downarrow}^{S}\left(p^{\mu}\right)\right]\right\}+ \\
& +c_{6}\left\{\frac{i N}{4 \sqrt{2} m}\left[\bar{\lambda}_{\uparrow}^{S}\left(p^{\mu}\right) \sigma^{0 i} \lambda_{\uparrow}^{A}\left(p^{\mu}\right)+\bar{\lambda}_{\downarrow}^{S}\left(p^{\mu}\right) \sigma^{0 i} \lambda_{\downarrow}^{A}\left(p^{\mu}\right)\right]-\right.
\end{aligned}
$$




$$
\begin{aligned}
& \left.-\frac{N}{8 \sqrt{2} m} \epsilon^{i j k}\left[\bar{\lambda}_{\uparrow}^{S}\left(p^{\mu}\right) \sigma^{j k} \lambda_{\uparrow}^{A}\left(p^{\mu}\right)-\bar{\lambda}_{\downarrow}^{S}\left(p^{\mu}\right) \sigma^{j k} \lambda_{\downarrow}^{A}\left(p^{\mu}\right)\right]\right\} \\
\mathbf{B}^{(+)}(\mathbf{p}, 0) & =\frac{i N}{2 m} \epsilon^{i j k}\left[c_{1} \bar{\lambda}_{\uparrow}^{S}\left(p^{\mu}\right) \sigma^{j k} \lambda_{\downarrow}^{S}\left(p^{\mu}\right)+c_{2} \bar{\lambda}_{\downarrow}^{S}\left(p^{\mu}\right) \sigma^{j k} \lambda_{\uparrow}^{S}\left(p^{\mu}\right)\right]- \\
& -\frac{N}{4 m}\left[c_{3} \bar{\lambda}_{\uparrow}^{S}\left(p^{\mu}\right) \sigma^{0 i} \lambda_{\downarrow}^{A}\left(p^{\mu}\right)+c_{4} \bar{\lambda}_{\downarrow}^{S}\left(p^{\mu}\right) \sigma^{0 i} \lambda_{\uparrow}^{A}\left(p^{\mu}\right)\right], \\
\mathbf{B}^{(+)}(\mathbf{p},+1) & =c_{5}\left\{\frac{-N}{4 \sqrt{2} m}\left[\bar{\lambda}_{\uparrow}^{S}\left(p^{\mu}\right) \sigma^{0 i} \lambda_{\uparrow}^{S}\left(p^{\mu}\right)+\bar{\lambda}_{\downarrow}^{S}\left(p^{\mu}\right) \sigma^{0 i} \lambda_{\downarrow}^{S}\left(p^{\mu}\right)\right]+\right. \\
& \left.+\frac{i N}{8 \sqrt{2} m} \epsilon^{i j k}\left[\bar{\lambda}_{\uparrow}^{S}\left(p^{\mu}\right) \sigma^{j k} \lambda_{\uparrow}^{S}\left(p^{\mu}\right)-\bar{\lambda}_{\downarrow}^{S}\left(p^{\mu}\right) \sigma^{j k} \lambda_{\downarrow}^{S}\left(p^{\mu}\right)\right]\right\}+ \\
& +c_{6}\left\{\frac{-N}{4 \sqrt{2} m}\left[\bar{\lambda}_{\uparrow}^{S}\left(p^{\mu}\right) \sigma^{0 i} \lambda_{\uparrow}^{A}\left(p^{\mu}\right)-\bar{\lambda}_{\downarrow}^{S}\left(p^{\mu}\right) \sigma^{0 i} \lambda_{\downarrow}^{A}\left(p^{\mu}\right)\right]+\right. \\
& \left.+\frac{i N}{8 \sqrt{2} m} \epsilon^{i j k}\left[\bar{\lambda}_{\uparrow}^{S}\left(p^{\mu}\right) \sigma^{j k} \lambda_{\uparrow}^{A}\left(p^{\mu}\right)+\bar{\lambda}_{\downarrow}^{S}\left(p^{\mu}\right) \sigma^{j k} \lambda_{\downarrow}^{A}\left(p^{\mu}\right)\right]\right\} \\
\mathbf{B}^{(+)}(\mathbf{p},-1) & =c_{5}\left\{\frac{-N}{4 \sqrt{2} m}\left[\bar{\lambda}_{\uparrow}^{S}\left(p^{\mu}\right) \sigma^{0 i} \lambda_{\uparrow}^{S}\left(p^{\mu}\right)+\bar{\lambda}_{\downarrow}^{S}\left(p^{\mu}\right) \sigma^{0 i} \lambda_{\downarrow}^{S}\left(p^{\mu}\right)\right]-\right. \\
& \left.-\frac{i N}{8 \sqrt{2} m} \epsilon^{i j k}\left[\bar{\lambda}_{\uparrow}^{S}\left(p^{\mu}\right) \sigma^{j k} \lambda_{\uparrow}^{S}\left(p^{\mu}\right)-\bar{\lambda}_{\downarrow}^{S}\left(p^{\mu}\right) \sigma^{j k} \lambda_{\downarrow}^{S}\left(p^{\mu}\right)\right]\right\}+ \\
& +c_{6}\left\{\frac{N}{4 \sqrt{2} m}\left[\bar{\lambda}_{\uparrow}^{S}\left(p^{\mu}\right) \sigma^{0 i} \lambda_{\uparrow}^{A}\left(p^{\mu}\right)-\bar{\lambda}_{\downarrow}^{S}\left(p^{\mu}\right) \sigma^{0 i} \lambda_{\downarrow}^{A}\left(p^{\mu}\right)\right]+\right. \\
& \left.\frac{i N}{8 \sqrt{2} m} \epsilon^{i j k}\left[\bar{\lambda}_{\uparrow}^{S}\left(p^{\mu}\right) \sigma^{j k} \lambda_{\uparrow}^{A}\left(p^{\mu}\right)+\bar{\lambda}_{\downarrow}^{S}\left(p^{\mu}\right) \sigma^{j k} \lambda_{\downarrow}^{A}\left(p^{\mu}\right)\right]\right\} \\
& \\
& \\
& \\
&
\end{aligned}
$$

We still apparently note that on using the formulas of the Appendix A (26a-26h) one can obtain formally different formulas connecting an antisymmetric tensor of the rank 2 and tensor currents composed of $\lambda_{\uparrow \downarrow}^{A, S}$ spinors. One can also repeat the derivation of the formulas of this paper on using the Dirac 4-spinors, see the formulas (18a $-18 \mathrm{~d})$, thus arriving at the electron-positron theory of light. We leave this simple exercise to the reader.

\section{WHERE MAY THE BEREZINSKY ARGUMENTS FAIL?}

In refs. [10,11,13,14, the McLennan-Case construct [7] has been developed considerably. We showed the theoretical possibility of existence of bi-orthonormalstates which have slightly different anticommutation relations comparing with the Dirac fermion. It was postulated

$$
\left[a_{\eta^{\prime}}\left(p^{\prime \mu}\right), a_{\eta}^{\dagger}\left(p^{\mu}\right)\right]_{ \pm}=(2 \pi)^{3} 2 p_{0} \delta^{3}\left(\mathbf{p}-\mathbf{p}^{\prime}\right) \delta_{\eta,-\eta^{\prime}}
$$




$$
\begin{aligned}
& {\left[b_{\eta^{\prime}}\left(p^{\prime \mu}\right), b_{\eta}^{\dagger}\left(p^{\mu}\right)\right]_{ \pm}=(2 \pi)^{3} 2 p_{0} \delta^{3}\left(\mathbf{p}-\mathbf{p}^{\prime}\right) \delta_{\eta,-\eta^{\prime}}} \\
& {\left[a_{\eta^{\prime}}\left(p^{\prime \mu}\right), b_{\eta}^{\dagger}\left(p^{\mu}\right)\right]_{ \pm}=0 .}
\end{aligned}
$$

for self/anti-self charge conjugate states. With taking into account these new ideas we re-examine the Berezynsky proof of the Price's theorem.

1) In ref. [5] (particularly in the first postulate, Eq. (6)) the author assumed neutrinos to be massless. In the massive case we have to use the generalized expressions (see, for instance, ref. [12, p. 177]) instead of (6) of ref. [5].

2) The author of ref. [5] assumed that neutrinos are fermions and (in his notation) the operators $a_{i}(\mathbf{k})$ and $b_{i}(\mathbf{k})$ obey the commutation relations

$$
\left[a_{i}^{\dagger}(\mathbf{k}), a_{j}\left(\mathbf{k}^{\prime}\right)\right]_{+}=\left[b_{i}^{\dagger}(\mathbf{k}), b_{j}\left(\mathbf{k}^{\prime}\right)\right]_{+}=\delta_{i j} \delta\left(\mathbf{k}-\mathbf{k}^{\prime}\right)
$$

If we assume that the neutrinos are bi-orthonormal states in the Berezinsky notation the analogues of anticommutation relations (5a-5c) may be expressed (in a particular case) as follows:

$$
\left[a_{i}^{\dagger}(\mathbf{k}), b_{j}\left(\mathbf{k}^{\prime}\right)\right]_{+}=\left[b_{i}^{\dagger}(\mathbf{k}), a_{j}\left(\mathbf{k}^{\prime}\right)\right]_{+}=e^{i \phi(i, j)} \delta_{i j} \delta\left(\mathbf{k}-\mathbf{k}^{\prime}\right)
$$

with indices $i$ and $j$ referring to " $i$-th" or " $j$-th" (anti)neutrino, respectively. All other anticommutators are equal to zero.

3) The right- and left- circular polarized photons have been assumed there to be massless too. We still advocate that they also may be self/anti-self charge conjugate $\left(\right.$ or $\Gamma_{5} \mathcal{C}$ self/antiself charge conjugate) massive states [11.

4) As a consequence of the item (3) we suggested [15] two definitions of the linearpolarized radiation.

For field theorists it is known that the change of the polarization state of massive particles can be made by the boost (and/or other non-unitary operations). On the other hand, it appears that for $j=1$ states (relevant to the problem at hand) the change of polarization can be made by means of the change of the basis of the corresponding complex vector space, 
i. e. by the rotation (on the classical level). It is produced by an unitary matrix. For instance, if one describes the magnetic field as

$$
\mathbf{B}^{\text {circ. }}=\frac{B^{(0)}}{\sqrt{2}}\left[\left(\begin{array}{l}
i \\
1 \\
0
\end{array}\right) e^{+i \phi}+\left(\begin{array}{c}
-i \\
1 \\
0
\end{array}\right) e^{-i \phi}\right],
$$

$(\phi=\omega t-\mathbf{k} \cdot \mathbf{r})$ on using the unitary matrix

$$
U=\frac{1}{\sqrt{2}}\left(\begin{array}{ccc}
-i & 1 & 0 \\
i & 1 & 0 \\
0 & 0 & \sqrt{2}
\end{array}\right)
$$

one can obtain the linear polarized (in the plane $X Y$ ) radiationf.

${ }^{2}$ If one wishes to see the real-valued magnetic fields instead of phasors here they are:

$$
\mathbf{B}_{x}^{\text {circ. }}=-\sqrt{2} B^{(0)} \sin \phi \quad, \mathbf{B}_{y}^{\text {circ. }}=+\sqrt{2} B^{(0)} \cos \phi \quad .
$$

or

$$
\mathbf{B}_{x}^{\text {lin. }}=+B^{(0)} \cos \phi \quad, \mathbf{B}_{y}^{\text {lin. }}=+B^{(0)} \cos \phi,
$$

i. e., in the latter case one obtains the linear polarized radiation with the polarization angle equal to $\pi / 4$. Of course, the given unitary matrix can be easily generalized to account for other polarization angles.

${ }^{3}$ The transformation of transverse components with the matrix $L$ used by G. Hunter 16, Eq.(19)] is not generally unitary:

$$
L \sim\left(\begin{array}{ccc}
(A-B) \cos \alpha & -(A+B) \sin \alpha & 0 \\
(A-B) \sin \alpha & (A+B) \cos \alpha & 0 \\
0 & 0 & 1
\end{array}\right),
$$

with $\alpha$ being the polar angle of the cylindrical system of coordinates. In the case of the linear polarization defined in such a way [16] one has $\mathbf{B} \times \mathbf{B}^{*}=0$. This transformation may also change the normalization of the corresponding vectors which in the quantized case correspond to a particle 


$$
\mathbf{B}^{\text {lin. }}=U \mathbf{B}^{\text {circ. }}=B^{(0)}\left[\left(\begin{array}{l}
1 \\
0 \\
0
\end{array}\right) e^{+i \phi}+\left(\begin{array}{l}
0 \\
1 \\
0
\end{array}\right) e^{-i \phi}\right] .
$$

Of course, the corresponding quantum covering of the above transformation will be different from (9) of ref. [5].

5) Furthermore, in private communication in January, 1999 Prof. W. Perkins pointed out [17 that the requirement of what Berezinsky considered as a condition of "genuinely neutrality" for a photon (the condition 5 of Berezinsky, Eqs. $(10,11)$ of ref. [5]) may be lifted (see also $[6 b])$.

\section{DISCUSSION AND CONCLUSION}

In the papers of Barut, e.g., ref. [18] a self-field formulation of quantum electrodynamics have been proposed. It is based on the use of the solution

$$
\mathcal{A}^{\mu}(x)=\int d^{4} y D^{\mu \nu}(x-y) j_{\nu}(y)
$$

of the coupled Maxwell-Dirac equation

$$
\partial^{\mu} F_{\mu \nu}(x)=e \bar{\Psi}(x) \gamma_{\nu} \Psi(x)
$$

$D_{\mu \nu}(x-y)$ is a Green's function of electromagnetic field in the usual potential formulation. In a series of the works A. Barut et al. have shown that this formulation of quantum electrodynamics (based on the iteration procedure, not on the perturbation theory) leads to the same experimental predictions as the ordinary formalism.

and an anti-particle. The determinant of the transformation is, in general, not equal to the unit. While the determinant of our matrix is also not equal to the unit $(\operatorname{det} U=-i)$, but the norm of the corresponding quantum states is still preserved (while this is not so for the corresponding real quantities). 
Let me try to write the formula (13) in the momentum space (To my knowledge, such attempts are absent in the literature). I consider momenta as $q=\lambda t$ and $p=(\lambda-1) t, \lambda$ is some function spanned from 0 to 1 . In this case

$$
\begin{aligned}
\mathcal{A}_{\mu}(x) & =-e \int \frac{d^{3} \mathbf{p} d^{3} \mathbf{q}}{(2 \pi)^{6}} \frac{D_{F}\left((q-p)^{2}\right)}{2 m \sqrt{E_{p} E_{q}}} \sum_{\sigma \sigma^{\prime}}\left\{\bar{u}_{\sigma}(\mathbf{p}) \gamma^{\mu} u_{\sigma^{\prime}}(\mathbf{q}) e^{i(q-p) x} a_{\sigma}^{\dagger}(\mathbf{p}) a_{\sigma^{\prime}}(\mathbf{q})+\right. \\
& \left.+\bar{v}_{\sigma}(\mathbf{p}) \gamma^{\mu} v_{\sigma^{\prime}}(\mathbf{q}) e^{-i(q-p) x} b_{\sigma}(\mathbf{p}) b_{\sigma^{\prime}}^{\dagger}(\mathbf{q})\right\} \quad,
\end{aligned}
$$

and, hence,

$$
\mathcal{A}_{\mu}(t)=\int_{0}^{1} d \lambda f\left(\lambda, t^{2}\right) \sum_{\sigma \sigma^{\prime}, \pm} \bar{\psi}_{\sigma}^{ \pm}((\lambda-1) t) \gamma^{\mu} \psi_{\sigma^{\prime}}^{ \pm}(\lambda t)
$$

Surprisingly, you may see the well-known Jordan ansatz. Thus, referring to the remark of the previous paragraph one can state the longitudinal de Broglie-Jordan-Barut potential can describe quantumelectrodynamic processes sufficiently good.

We think that in order to describe transverse components of the 4 -vector potential (leftand right- polarized radiations) correctly one should set up the different commutation relations for the 4-spinor fields which are different from those used in the Dirac theory. It is also possible that in order to overcome dificulties related to teh Pryce theorem one should use the generalized definition of linear polarized radiation. In general, the room of choosing the corresponding constants in the superpositions permits one to obtain various types of (anti)commutation relations for the composite particles Tensor currents can also be generalzied. In my opinion, resulting expressions of the type $F_{\lambda}^{\mu \nu} \sim \bar{\nu}_{\eta}\left(\sigma^{\mu \nu} \pm i \widetilde{\sigma}^{\mu \nu}\right) \nu_{\eta^{\prime}}$ (and its dual conjugates) also permit to construct the neutrino theory of light.

Acknowledgments. The work was motivated by very useful frank discussions and phone conversations with Prof. A. F. Pashkov during last 15 years. I acknowledge email communications from Profs. D. V. Ahluwalia, V. Berezinsky, D. Bernard, B. Fauser, W. Perkins and J. R. Zeni.

Zacatecas University, México, is thanked for awarding the full professorship. This work has been partly supported by the Mexican Sistema Nacional de Investigadores. 


\section{APPENDIX A}

Explicit forms of the second-type 4-spinors, refs. 10,11,13 in the Weyl representation are (the spinorial basis is fixed as in ref. [13], cf. [14, 19]):

$$
\begin{aligned}
& \lambda_{\uparrow}^{S}\left(p^{\mu}\right)=+i \rho_{\downarrow}^{A}\left(p^{\mu}\right)=\frac{1}{2 \sqrt{p_{0}+m}}\left(\begin{array}{c}
i p_{l} \\
i\left(p^{-}+m\right) \\
p^{-}+m \\
-p_{r}
\end{array}\right)\left(\begin{array}{c}
-i p_{l} \\
-i\left(p^{-}+m\right) \\
p^{-}+m \\
-p_{r}
\end{array}\right) \\
& \lambda_{\uparrow}^{A}\left(p^{\mu}\right)=-i \rho_{\downarrow}^{S}\left(p^{\mu}\right)=\frac{1}{2 \sqrt{p_{0}+m}}\left(\begin{array}{c}
-i\left(p^{+}+m\right) \\
-i p_{r} \\
-p_{l} \\
\lambda_{\downarrow}^{S}\left(p^{\mu}\right)=-i \rho_{\uparrow}^{A}\left(p^{\mu}\right)=\frac{1}{2 \sqrt{p_{0}+m}} \\
\lambda_{\downarrow}^{A}\left(p^{\mu}\right)=+i \rho_{\uparrow}^{S}\left(p^{\mu}\right)=\frac{1}{2 \sqrt{p_{0}+m}} \\
p^{+}+m
\end{array}\right), \\
&\left(\begin{array}{c}
i\left(p^{+}+m\right) \\
i p_{r} \\
-p_{l} \\
p^{+}+m
\end{array}\right)
\end{aligned}
$$

Their connections with the Dirac 4-spinors are the following:

$$
\begin{aligned}
& \lambda_{\uparrow}^{S}\left(p^{\mu}\right)=+i \frac{1+\gamma^{5}}{2} u_{\downarrow}\left(p^{\mu}\right)+\frac{1-\gamma^{5}}{2} u_{\uparrow}\left(p^{\mu}\right), \\
& \lambda_{\downarrow}^{S}\left(p^{\mu}\right)==-i \frac{1+\gamma^{5}}{2} u_{\uparrow}\left(p^{\mu}\right)+\frac{1-\gamma^{5}}{2} u_{\downarrow}\left(p^{\mu}\right), \\
& \lambda_{\uparrow}^{A}\left(p^{\mu}\right)==-i \frac{1+\gamma^{5}}{2} u_{\downarrow}\left(p^{\mu}\right)+\frac{1-\gamma^{5}}{2} u_{\uparrow}\left(p^{\mu}\right), \\
& \lambda_{\downarrow}^{A}\left(p^{\mu}\right)==+i \frac{1+\gamma^{5}}{2} u_{\uparrow}\left(p^{\mu}\right)+\frac{1-\gamma^{5}}{2} u_{\downarrow}\left(p^{\mu}\right)
\end{aligned}
$$

Normalization conditions read: 


$$
\begin{array}{ll}
\bar{\lambda}_{\uparrow}^{S}\left(p^{\mu}\right) \lambda_{\downarrow}^{S}\left(p^{\mu}\right)=-i m, & \bar{\lambda}_{\downarrow}^{S}\left(p^{\mu}\right) \lambda_{\uparrow}^{S}\left(p^{\mu}\right)=+i m \\
\bar{\lambda}_{\uparrow}^{A}\left(p^{\mu}\right) \lambda_{\downarrow}^{A}\left(p^{\mu}\right)=+i m, & \bar{\lambda}_{\downarrow}^{A}\left(p^{\mu}\right) \lambda_{\uparrow}^{A}\left(p^{\mu}\right)=-i m
\end{array}
$$

All other products for $\lambda$ - spinors are equal to zero.

From (19a,19b) one deduces:

$$
\begin{array}{ll}
\bar{\lambda}_{\uparrow}^{S}\left(p^{\mu}\right) \gamma^{5} \lambda_{\downarrow}^{A}\left(p^{\mu}\right)=+i m, & \bar{\lambda}_{\downarrow}^{S}\left(p^{\mu}\right) \gamma^{5} \lambda_{\uparrow}^{A}\left(p^{\mu}\right)=-i m . \\
\bar{\lambda}_{\uparrow}^{A}\left(p^{\mu}\right) \gamma^{5} \lambda_{\downarrow}^{S}\left(p^{\mu}\right)=-i m, & \bar{\lambda}_{\downarrow}^{A}\left(p^{\mu}\right) \gamma^{5} \lambda_{\uparrow}^{S}\left(p^{\mu}\right)=+i m .
\end{array}
$$

All other products for $\lambda$ - spinors are equal to zero.

Vector currents are:f

$$
\begin{aligned}
& \bar{\lambda}_{\uparrow}^{S}\left(p^{\mu}\right) \gamma^{\mu} \lambda_{\uparrow}^{S}\left(p^{\mu}\right)=\left(\begin{array}{c}
p^{-} \\
p_{1}-\frac{p_{1} p_{3}}{p_{0}+m} \\
p_{2}-\frac{p_{2} p_{3}}{p_{0}+m} \\
p_{3}-m-\frac{p_{3}^{2}}{p_{0}+m}
\end{array}\right), \quad \bar{\lambda}_{\uparrow}^{S}\left(p^{\mu}\right) \gamma^{\mu} \lambda_{\downarrow}^{S}\left(p^{\mu}\right)=-\left(\begin{array}{c}
p_{1} \\
m+\frac{p_{1}^{2}}{p_{0}+m} \\
\frac{p_{1} p_{2}}{p_{0}+m} \\
\frac{p_{1} p_{3}}{p_{0}+m}
\end{array}\right), \\
& \bar{\lambda}_{\uparrow}^{S}\left(p^{\mu}\right) \gamma^{\mu} \lambda_{\uparrow}^{A}\left(p^{\mu}\right)=\left(\begin{array}{l}
0 \\
0 \\
0 \\
0
\end{array}\right), \quad \bar{\lambda}_{\uparrow}^{S}\left(p^{\mu}\right) \gamma^{\mu} \lambda_{\downarrow}^{A}\left(p^{\mu}\right)=+i\left(\begin{array}{c}
p_{2} \\
\frac{p_{1} p_{2}}{p_{0}+m} \\
m+\frac{p_{2}^{2}}{p_{0}+m} \\
\frac{p_{2} p_{3}}{p_{0}+m}
\end{array}\right), \\
& \bar{\lambda}_{\downarrow}^{S}\left(p^{\mu}\right) \gamma^{\mu} \lambda_{\downarrow}^{S}\left(p^{\mu}\right)=\left(\begin{array}{c}
p^{+} \\
p_{1}+\frac{p_{1} p_{3}}{p_{0}+m} \\
p_{2}+\frac{p_{2} p_{3}}{p_{0}+m} \\
p_{3}+m+\frac{p_{3}^{2}}{p_{0}+m}
\end{array}\right), \quad \bar{\lambda}_{\downarrow}^{S}\left(p^{\mu}\right) \gamma^{\mu} \lambda_{\uparrow}^{S}\left(p^{\mu}\right)=-\left(\begin{array}{c}
p_{1} \\
m+\frac{p_{1}^{2}}{p_{0}+m} \\
\frac{p_{1} p_{2}}{p_{0}+m} \\
\frac{p_{1} p_{3}}{p_{0}+m}
\end{array}\right), \\
& \bar{\lambda}_{\downarrow}^{S}\left(p^{\mu}\right) \gamma^{\mu} \lambda_{\downarrow}^{A}\left(p^{\mu}\right)=\left(\begin{array}{c}
0 \\
0 \\
0 \\
0
\end{array}\right), \quad \bar{\lambda}_{\downarrow}^{S}\left(p^{\mu}\right) \gamma^{\mu} \lambda_{\uparrow}^{A}\left(p^{\mu}\right)=-i\left(\begin{array}{c}
p_{2} \\
\frac{p_{1} p_{2}}{p_{0}+m} \\
m+\frac{p_{2}^{2}}{p_{0}+m} \\
\frac{p_{2} p_{3}}{p_{0}+m}
\end{array}\right) ;
\end{aligned}
$$

${ }^{4}$ We imply that the first element corresponds to $\mu=0$ and, subsequently, $\mu=1,2,3$. 
and, hence,

$$
\begin{aligned}
& \bar{\lambda}_{\uparrow}^{A}\left(p^{\mu}\right) \gamma^{\mu} \lambda_{\uparrow}^{S}\left(p^{\mu}\right)=0, \\
& \bar{\lambda}_{\uparrow}^{A}\left(p^{\mu}\right) \gamma^{\mu} \lambda_{\downarrow}^{S}\left(p^{\mu}\right)=\bar{\lambda}_{\uparrow}^{S}\left(p^{\mu}\right) \gamma^{\mu} \lambda_{\downarrow}^{A}\left(p^{\mu}\right), \\
& \bar{\lambda}_{\uparrow}^{A}\left(p^{\mu}\right) \gamma^{\mu} \lambda_{\uparrow}^{A}\left(p^{\mu}\right)=\bar{\lambda}_{\uparrow}^{S}\left(p^{\mu}\right) \gamma^{\mu} \lambda_{\uparrow}^{S}\left(p^{\mu}\right), \\
& \bar{\lambda}_{\uparrow}^{A}\left(p^{\mu}\right) \gamma^{\mu} \lambda_{\downarrow}^{A}\left(p^{\mu}\right)=\bar{\lambda}_{\uparrow}^{S}\left(p^{\mu}\right) \gamma^{\mu} \lambda_{\downarrow}^{S}\left(p^{\mu}\right), \\
& \bar{\lambda}_{\downarrow}^{A}\left(p^{\mu}\right) \gamma^{\mu} \lambda_{\downarrow}^{S}\left(p^{\mu}\right)=0, \\
& \bar{\lambda}_{\downarrow}^{A}\left(p^{\mu}\right) \gamma^{\mu} \lambda_{\uparrow}^{S}\left(p^{\mu}\right)=\bar{\lambda}_{\downarrow}^{S}\left(p^{\mu}\right) \gamma^{\mu} \lambda_{\uparrow}^{A}\left(p^{\mu}\right), \\
& \bar{\lambda}_{\downarrow}^{A}\left(p^{\mu}\right) \gamma^{\mu} \lambda_{\uparrow}^{A}\left(p^{\mu}\right)=\bar{\lambda}_{\downarrow}^{S}\left(p^{\mu}\right) \gamma^{\mu} \lambda_{\uparrow}^{S}\left(p^{\mu}\right), \\
& \bar{\lambda}_{\downarrow}^{A}\left(p^{\mu}\right) \gamma^{\mu} \lambda_{\downarrow}^{A}\left(p^{\mu}\right)=\bar{\lambda}_{\downarrow}^{S}\left(p^{\mu}\right) \gamma^{\mu} \lambda_{\downarrow}^{S}\left(p^{\mu}\right),
\end{aligned}
$$

Axial-vector currents are:

$$
\begin{aligned}
& \bar{\lambda}_{\uparrow}^{S}\left(p^{\mu}\right) \gamma^{\mu} \gamma^{5} \lambda_{\uparrow}^{S}\left(p^{\mu}\right)=\left(\begin{array}{c}
0 \\
0 \\
0 \\
0
\end{array}\right), \quad \bar{\lambda}_{\uparrow}^{S}\left(p^{\mu}\right) \gamma^{\mu} \gamma^{5} \lambda_{\downarrow}^{S}\left(p^{\mu}\right)=-i\left(\begin{array}{c}
p_{2} \\
\frac{p_{1} p_{2}}{p_{0}+m} \\
m+\frac{p_{2}^{2}}{p_{0}+m} \\
\frac{p_{2} p_{3}}{p_{0}+m}
\end{array}\right), \\
& \bar{\lambda}_{\uparrow}^{S}\left(p^{\mu}\right) \gamma^{\mu} \gamma^{5} \lambda_{\uparrow}^{A}\left(p^{\mu}\right)=-\left(\begin{array}{c}
p^{-} \\
p_{1}-\frac{p_{1} p_{3}}{p_{0}+m} \\
p_{2}-\frac{p_{2} p_{3}}{p_{0}+m} \\
p_{3}-m-\frac{p_{3}^{2}}{p_{0}+m}
\end{array}\right), \quad \bar{\lambda}_{\uparrow}^{S}\left(p^{\mu}\right) \gamma^{\mu} \gamma^{5} \lambda_{\downarrow}^{A}\left(p^{\mu}\right)=\left(\begin{array}{c}
p_{1} \\
m+\frac{p_{1}^{2}}{p_{0}+m} \\
\frac{p_{1} p_{2}}{p_{0}+m} \\
\frac{p_{1} p_{3}}{p_{0}+m}
\end{array}\right), \\
& \bar{\lambda}_{\downarrow}^{S}\left(p^{\mu}\right) \gamma^{\mu} \gamma^{5} \lambda_{\downarrow}^{S}\left(p^{\mu}\right)=\left(\begin{array}{c}
0 \\
0 \\
0 \\
0
\end{array}\right), \quad \bar{\lambda}_{\downarrow}^{S}\left(p^{\mu}\right) \gamma^{\mu} \gamma^{5} \lambda_{\uparrow}^{S}\left(p^{\mu}\right)=+i\left(\begin{array}{c}
p_{2} \\
\frac{p_{1} p_{2}}{p_{0}+m} \\
m+\frac{p_{2}^{2}}{p_{0}+m} \\
\frac{p_{2} p_{3}}{p_{0}+m}
\end{array}\right) \\
& \bar{\lambda}_{\downarrow}^{S}\left(p^{\mu}\right) \gamma^{\mu} \gamma^{5} \lambda_{\uparrow}^{A}\left(p^{\mu}\right)=\left(\begin{array}{c}
p_{1} \\
m+\frac{p_{1}^{2}}{p_{0}+m} \\
\frac{p_{1} p_{2}}{p_{0}+m} \\
\frac{p_{1} p_{3}}{p_{0}+m}
\end{array}\right), \quad \bar{\lambda}_{\downarrow}^{S}\left(p^{\mu}\right) \gamma^{\mu} \gamma^{5} \lambda_{\downarrow}^{A}\left(p^{\mu}\right)=-\left(\begin{array}{c}
p^{+} \\
p_{1}+\frac{p_{1} p_{3}}{p_{0}+m} \\
p_{2}+\frac{p_{2} p_{3}}{p_{0}+m} \\
m+\frac{p_{3}^{2}}{p_{0}+m}
\end{array}\right) ;
\end{aligned}
$$


and, hence,

$$
\begin{aligned}
& \bar{\lambda}_{\uparrow}^{A}\left(p^{\mu}\right) \gamma^{\mu} \gamma^{5} \lambda_{\uparrow}^{S}\left(p^{\mu}\right)=\bar{\lambda}_{\uparrow}^{S}\left(p^{\mu}\right) \gamma^{\mu} \gamma^{5} \lambda_{\uparrow}^{A}\left(p^{\mu}\right), \\
& \bar{\lambda}_{\uparrow}^{A}\left(p^{\mu}\right) \gamma^{\mu} \gamma^{5} \lambda_{\downarrow}^{S}\left(p^{\mu}\right)=\bar{\lambda}_{\uparrow}^{S}\left(p^{\mu}\right) \gamma^{\mu} \gamma^{5} \lambda_{\downarrow}^{A}\left(p^{\mu}\right), \\
& \bar{\lambda}_{\uparrow}^{A}\left(p^{\mu}\right) \gamma^{\mu} \gamma^{5} \lambda_{\uparrow}^{A}\left(p^{\mu}\right)=0, \\
& \bar{\lambda}_{\uparrow}^{A}\left(p^{\mu}\right) \gamma^{\mu} \gamma^{5} \lambda_{\downarrow}^{A}\left(p^{\mu}\right)=\bar{\lambda}_{\uparrow}^{S}\left(p^{\mu}\right) \gamma^{\mu} \gamma^{5} \lambda_{\downarrow}^{S}\left(p^{\mu}\right), \\
& \bar{\lambda}_{\downarrow}^{A}\left(p^{\mu}\right) \gamma^{\mu} \gamma^{5} \lambda_{\downarrow}^{S}\left(p^{\mu}\right)=\bar{\lambda}_{\downarrow}^{S}\left(p^{\mu}\right) \gamma^{\mu} \gamma^{5} \lambda_{\downarrow}^{A}\left(p^{\mu}\right) \\
& \bar{\lambda}_{\downarrow}^{A}\left(p^{\mu}\right) \gamma^{\mu} \gamma^{5} \lambda_{\uparrow}^{S}\left(p^{\mu}\right)=\bar{\lambda}_{\downarrow}^{S}\left(p^{\mu}\right) \gamma^{\mu} \gamma^{5} \lambda_{\uparrow}^{A}\left(p^{\mu}\right), \\
& \bar{\lambda}_{\downarrow}^{A}\left(p^{\mu}\right) \gamma^{\mu} \gamma^{5} \lambda_{\downarrow}^{A}\left(p^{\mu}\right)=0, \\
& \bar{\lambda}_{\downarrow}^{A}\left(p^{\mu}\right) \gamma^{\mu} \gamma^{5} \lambda_{\uparrow}^{A}\left(p^{\mu}\right)=\bar{\lambda}_{\downarrow}^{S}\left(p^{\mu}\right) \gamma^{\mu} \gamma^{5} \lambda_{\uparrow}^{S}\left(p^{\mu}\right) .
\end{aligned}
$$

Finally, tensor currents are: ${ }^{5}$

$$
\begin{aligned}
& \bar{\lambda}_{\uparrow}^{S}\left(p^{\mu}\right) \sigma^{\mu \nu} \lambda_{\uparrow}^{S}\left(p^{\mu}\right)=\left(\begin{array}{c}
-p^{-}+\frac{p_{1}^{2}}{p_{0}+m} \\
\frac{p_{1} p_{2}}{p_{0}+m} \\
-p_{1}+\frac{p_{1} p_{3}}{p_{0}+m} \\
-\frac{p_{1} p_{2}}{p_{0}+m} \\
p^{-}-\frac{p_{2}^{2}}{p_{0}+m} \\
p_{2}-\frac{p_{2} p_{3}}{p_{0}+m}
\end{array}\right), \\
& \bar{\lambda}_{\uparrow}^{S}\left(p^{\mu}\right) \sigma^{\mu \nu} \lambda_{\downarrow}^{S}\left(p^{\mu}\right)=\bar{\lambda}_{\downarrow}^{S}\left(p^{\mu}\right) \sigma^{\mu \nu} \lambda_{\uparrow}^{S}\left(p^{\mu}\right)=\left(\begin{array}{c}
-\frac{p_{1} p_{3}}{p_{0}+m} \\
-\frac{p_{2} p_{3}}{p_{0}+m} \\
p_{0}-\frac{p_{3}^{2}}{p_{0}+m} \\
p_{2} \\
-p_{1} \\
0
\end{array}\right),
\end{aligned}
$$

\footnotetext{
${ }^{5}$ We imply that the first element corresponds to $\mu=0, \nu=1$, and, subsequently, $(\mu=0, \nu=2)$, $(\mu=0, \nu=3),(\mu=2, \nu=3),(\mu=3, \nu=1)(\mu=1, \nu=2)$.
} 


$$
\begin{aligned}
& \bar{\lambda}_{\uparrow}^{S}\left(p^{\mu}\right) \sigma^{\mu \nu} \lambda_{\uparrow}^{A}\left(p^{\mu}\right)=+i\left(\begin{array}{c}
\frac{p_{1} p_{2}}{p_{0}+m} \\
-p^{-}+\frac{p_{2}^{2}}{p_{0}+m} \\
-p_{2}+\frac{p_{2} p_{3}}{p_{0}+m} \\
-p^{-}+\frac{p_{1}^{2}}{p_{0}+m} \\
\frac{p_{1} p_{2}}{p_{0}+m} \\
-p_{1}+\frac{p_{1} p_{3}}{p_{0}+m}
\end{array}\right), \\
& \bar{\lambda}_{\uparrow}^{S}\left(p^{\mu}\right) \sigma^{\mu \nu} \lambda_{\downarrow}^{A}\left(p^{\mu}\right)=\bar{\lambda}_{\downarrow}^{S}\left(p^{\mu}\right) \sigma^{\mu \nu} \lambda_{\uparrow}^{A}\left(p^{\mu}\right)=+i\left(\begin{array}{c}
-p_{2} \\
p_{1} \\
0 \\
-\frac{p_{1} p_{3}}{p_{0}+m} \\
-\frac{p_{2} p_{3}}{p_{0}+m} \\
p_{0}-\frac{p_{3}^{2}}{p_{0}+m}
\end{array}\right), \\
& \bar{\lambda}_{\downarrow}^{S}\left(p^{\mu}\right) \sigma^{\mu \nu} \lambda_{\downarrow}^{S}\left(p^{\mu}\right)=-\left(\begin{array}{c}
-p^{+}+\frac{p_{1}^{2}}{p_{0}+m} \\
\frac{p_{1} p_{2}}{p_{0}+m} \\
p_{1}+\frac{p_{1} p_{3}}{p_{0}+m} \\
\frac{p_{1} p_{2}}{p_{0}+m} \\
-p^{+}+\frac{p_{2}^{2}}{p_{0}+m} \\
p_{2}+\frac{p_{2} p_{3}}{p_{0}+m}
\end{array}\right) \\
& \bar{\lambda}_{\downarrow}^{S}\left(p^{\mu}\right) \sigma^{\mu \nu} \lambda_{\downarrow}^{A}\left(p^{\mu}\right)=+i\left(\begin{array}{c}
\frac{p_{1} p_{2}}{p_{0}+m} \\
-p^{+}+\frac{p_{2}^{2}}{p_{0}+m} \\
p_{2}+\frac{p_{2} p_{3}}{p_{0}+m} \\
p^{+}-\frac{p_{1}^{2}}{p_{0}+m} \\
-\frac{p_{1} p_{2}}{p_{0}+m} \\
-p_{1}-\frac{p_{1} p_{3}}{p_{0}+m}
\end{array}\right) \text {. }
\end{aligned}
$$

and, hence,

$$
\begin{aligned}
& \bar{\lambda}_{\uparrow}^{A}\left(p^{\mu}\right) \sigma^{\mu \nu} \lambda_{\uparrow}^{S}\left(p^{\mu}\right)=-\bar{\lambda}_{\uparrow}^{S}\left(p^{\mu}\right) \sigma^{\mu \nu} \lambda_{\uparrow}^{A}\left(p^{\mu}\right), \\
& \bar{\lambda}_{\uparrow}^{A}\left(p^{\mu}\right) \sigma^{\mu \nu} \lambda_{\downarrow}^{S}\left(p^{\mu}\right)=-\bar{\lambda}_{\uparrow}^{S}\left(p^{\mu}\right) \sigma^{\mu \nu} \lambda_{\downarrow}^{A}\left(p^{\mu}\right), \\
& \bar{\lambda}_{\uparrow}^{A}\left(p^{\mu}\right) \sigma^{\mu \nu} \lambda_{\uparrow}^{A}\left(p^{\mu}\right)=-\bar{\lambda}_{\uparrow}^{S}\left(p^{\mu}\right) \sigma^{\mu \nu} \lambda_{\uparrow}^{S}\left(p^{\mu}\right),
\end{aligned}
$$




$$
\begin{aligned}
& \bar{\lambda}_{\uparrow}^{A}\left(p^{\mu}\right) \sigma^{\mu \nu} \lambda_{\downarrow}^{A}\left(p^{\mu}\right)=-\bar{\lambda}_{\uparrow}^{S}\left(p^{\mu}\right) \sigma^{\mu \nu} \lambda_{\downarrow}^{S}\left(p^{\mu}\right), \\
& \bar{\lambda}_{\downarrow}^{A}\left(p^{\mu}\right) \sigma^{\mu \nu} \lambda_{\uparrow}^{S}\left(p^{\mu}\right)=-\bar{\lambda}_{\downarrow}^{S}\left(p^{\mu}\right) \sigma^{\mu \nu} \lambda_{\uparrow}^{A}\left(p^{\mu}\right), \\
& \bar{\lambda}_{\downarrow}^{A}\left(p^{\mu}\right) \sigma^{\mu \nu} \lambda_{\downarrow}^{S}\left(p^{\mu}\right)=-\bar{\lambda}_{\downarrow}^{S}\left(p^{\mu}\right) \sigma^{\mu \nu} \lambda_{\downarrow}^{A}\left(p^{\mu}\right), \\
& \bar{\lambda}_{\downarrow}^{A}\left(p^{\mu}\right) \sigma^{\mu \nu} \lambda_{\uparrow}^{A}\left(p^{\mu}\right)=-\bar{\lambda}_{\downarrow}^{S}\left(p^{\mu}\right) \sigma^{\mu \nu} \lambda_{\uparrow}^{S}\left(p^{\mu}\right), \\
& \bar{\lambda}_{\downarrow}^{A}\left(p^{\mu}\right) \sigma^{\mu \nu} \lambda_{\downarrow}^{A}\left(p^{\mu}\right)=-\bar{\lambda}_{\downarrow}^{S}\left(p^{\mu}\right) \sigma^{\mu \nu} \lambda_{\downarrow}^{S}\left(p^{\mu}\right),
\end{aligned}
$$

One can write corresponding relations between currents composed from the first-type spinors and 4-vector potentials and electromagnetic strengths. They are found either by direct calculations or by the application of the formulas of this Appendix.

Normalizations of the Dirac spinors (see the explicit forms in [20, p.53] $]^{6}$ ) are

$$
\begin{array}{rc}
\bar{u}_{\uparrow}\left(p^{\mu}\right) u_{\uparrow}\left(p^{\mu}\right)=m & \bar{u}_{\downarrow}\left(p^{\mu}\right) u_{\downarrow}\left(p^{\mu}\right)=m, \\
\bar{v}_{\uparrow}\left(p^{\mu}\right) v_{\uparrow}\left(p^{\mu}\right)=-m & \bar{v}_{\downarrow}\left(p^{\mu}\right) v_{\downarrow}\left(p^{\mu}\right)=-m .
\end{array}
$$

Therefore, one has

$$
\begin{array}{r}
\bar{u}_{\uparrow}\left(p^{\mu}\right) \gamma^{5} v_{\uparrow}\left(p^{\mu}\right)=m \quad \bar{u}_{\downarrow}\left(p^{\mu}\right) \gamma^{5} v_{\downarrow}\left(p^{\mu}\right)=m, \\
\bar{v}_{\uparrow}\left(p^{\mu}\right) \gamma^{5} u_{\uparrow}\left(p^{\mu}\right)=-m \quad \bar{v}_{\downarrow}\left(p^{\mu}\right) \gamma^{5} u_{\downarrow}\left(p^{\mu}\right)=-m ;
\end{array}
$$

and

$$
\begin{gathered}
\bar{u}_{\uparrow}\left(p^{\mu}\right) \gamma^{\mu} u_{\uparrow}\left(p^{\mu}\right)=\bar{u}_{\downarrow}\left(p^{\mu}\right) \gamma^{\mu} u_{\downarrow}\left(p^{\mu}\right)= \\
=\bar{u}_{\uparrow}\left(p^{\mu}\right) \gamma^{\mu} \gamma^{5} v_{\uparrow}\left(p^{\mu}\right)=\bar{u}_{\downarrow}\left(p^{\mu}\right) \gamma^{\mu} \gamma^{5} v_{\downarrow}\left(p^{\mu}\right)=\frac{m}{N} u^{\mu}\left(\mathbf{p}, 0_{t}\right), \\
\bar{u}_{\uparrow}\left(p^{\mu}\right) \gamma^{\mu} v_{\uparrow}\left(p^{\mu}\right)=-\bar{u}_{\downarrow}\left(p^{\mu}\right) \gamma^{\mu} v_{\downarrow}\left(p^{\mu}\right)= \\
=\bar{u}_{\uparrow}\left(p^{\mu}\right) \gamma^{\mu} \gamma^{5} u_{\uparrow}\left(p^{\mu}\right)=-\bar{u}_{\downarrow}\left(p^{\mu}\right) \gamma^{\mu} \gamma^{5} u_{\downarrow}\left(p^{\mu}\right)=\frac{m}{N} u^{\mu}(\mathbf{p}, 0), \\
\bar{u}_{\uparrow}\left(p^{\mu}\right) \gamma^{\mu} u_{\downarrow}\left(p^{\mu}\right)=\bar{u}_{\downarrow}\left(p^{\mu}\right) \gamma^{\mu} u_{\uparrow}\left(p^{\mu}\right)=\bar{u}_{\uparrow}\left(p^{\mu}\right) \gamma^{\mu} \gamma^{5} v_{\downarrow}\left(p^{\mu}\right)=\bar{u}_{\downarrow}\left(p^{\mu}\right) \gamma^{\mu} \gamma^{5} v_{\uparrow}\left(p^{\mu}\right)=0,
\end{gathered}
$$

\footnotetext{
${ }^{6}$ Those 4 -spinors were given in the standard representation. Please pay attention to misprints in formulas $(2.137,2.138)$ of ref. [20], $\frac{E+m}{2 m} \Rightarrow \sqrt{\frac{E+m}{2 m}}$.
} 


$$
\begin{aligned}
& \bar{u}_{\uparrow}\left(p^{\mu}\right) \gamma^{\mu} v_{\downarrow}\left(p^{\mu}\right)=\bar{u}_{\uparrow}\left(p^{\mu}\right) \gamma^{\mu} \gamma^{5} u_{\downarrow}\left(p^{\mu}\right)=\frac{m \sqrt{2}}{N} u^{\mu}(\mathbf{p},-1), \\
& \bar{u}_{\downarrow}\left(p^{\mu}\right) \gamma^{\mu} v_{\uparrow}\left(p^{\mu}\right)=\bar{u}_{\downarrow}\left(p^{\mu}\right) \gamma^{\mu} u_{\uparrow}\left(p^{\mu}\right)=-\frac{m \sqrt{2}}{N} u^{\mu}(\mathbf{p},+1) .
\end{aligned}
$$

One also deduces

$$
\begin{aligned}
& \bar{v}_{\uparrow}\left(p^{\mu}\right) \gamma^{\mu} u_{\uparrow}\left(p^{\mu}\right)=\bar{u}_{\uparrow}\left(p^{\mu}\right) \gamma^{\mu} v_{\uparrow}\left(p^{\mu}\right), \\
& \bar{v}_{\uparrow}\left(p^{\mu}\right) \gamma^{\mu} u_{\downarrow}\left(p^{\mu}\right)=\bar{u}_{\uparrow}\left(p^{\mu}\right) \gamma^{\mu} v_{\downarrow}\left(p^{\mu}\right), \\
& \bar{v}_{\uparrow}\left(p^{\mu}\right) \gamma^{\mu} v_{\uparrow}\left(p^{\mu}\right)=\bar{u}_{\uparrow}\left(p^{\mu}\right) \gamma^{\mu} u_{\uparrow}\left(p^{\mu}\right), \\
& \bar{v}_{\uparrow}\left(p^{\mu}\right) \gamma^{\mu} v_{\downarrow}\left(p^{\mu}\right)=\bar{u}_{\uparrow}\left(p^{\mu}\right) \gamma^{\mu} u_{\downarrow}\left(p^{\mu}\right)=0, \\
& \bar{v}_{\downarrow}\left(p^{\mu}\right) \gamma^{\mu} u_{\uparrow}\left(p^{\mu}\right)=\bar{u}_{\downarrow}\left(p^{\mu}\right) \gamma^{\mu} v_{\uparrow}\left(p^{\mu}\right), \\
& \bar{v}_{\downarrow}\left(p^{\mu}\right) \gamma^{\mu} u_{\downarrow}\left(p^{\mu}\right)=\bar{u}_{\downarrow}\left(p^{\mu}\right) \gamma^{\mu} v_{\downarrow}\left(p^{\mu}\right), \\
& \bar{v}_{\downarrow}\left(p^{\mu}\right) \gamma^{\mu} v_{\uparrow}\left(p^{\mu}\right)=\bar{u}_{\downarrow}\left(p^{\mu}\right) \gamma^{\mu} u_{\uparrow}\left(p^{\mu}\right)=0, \\
& \bar{v}_{\downarrow}\left(p^{\mu}\right) \gamma^{\mu} v_{\downarrow}\left(p^{\mu}\right)=\bar{u}_{\downarrow}\left(p^{\mu}\right) \gamma^{\mu} u_{\downarrow}\left(p^{\mu}\right) ;
\end{aligned}
$$

and

$$
\begin{aligned}
& \bar{v}_{\uparrow}\left(p^{\mu}\right) \gamma^{\mu} \gamma^{5} u_{\uparrow}\left(p^{\mu}\right)=\bar{u}_{\uparrow}\left(p^{\mu}\right) \gamma^{\mu} \gamma^{5} v_{\uparrow}\left(p^{\mu}\right), \\
& \bar{v}_{\uparrow}\left(p^{\mu}\right) \gamma^{\mu} \gamma^{5} u_{\downarrow}\left(p^{\mu}\right)=\bar{u}_{\uparrow}\left(p^{\mu}\right) \gamma^{\mu} \gamma^{5} v_{\downarrow}\left(p^{\mu}\right)=0, \\
& \bar{v}_{\uparrow}\left(p^{\mu}\right) \gamma^{\mu} \gamma^{5} v_{\uparrow}\left(p^{\mu}\right)=\bar{u}_{\uparrow}\left(p^{\mu}\right) \gamma^{\mu} \gamma^{5} u_{\uparrow}\left(p^{\mu}\right), \\
& \bar{v}_{\uparrow}\left(p^{\mu}\right) \gamma^{\mu} \gamma^{5} v_{\downarrow}\left(p^{\mu}\right)=\bar{u}_{\uparrow}\left(p^{\mu}\right) \gamma^{\mu} \gamma^{5} u_{\downarrow}\left(p^{\mu}\right), \\
& \bar{v}_{\downarrow}\left(p^{\mu}\right) \gamma^{\mu} \gamma^{5} u_{\uparrow}\left(p^{\mu}\right)=\bar{u}_{\downarrow}\left(p^{\mu}\right) \gamma^{\mu} \gamma^{5} v_{\uparrow}\left(p^{\mu}\right)=0, \\
& \bar{v}_{\downarrow}\left(p^{\mu}\right) \gamma^{\mu} \gamma^{5} u_{\downarrow}\left(p^{\mu}\right)=\bar{u}_{\downarrow}\left(p^{\mu}\right) \gamma^{\mu} \gamma^{5} v_{\downarrow}\left(p^{\mu}\right), \\
& \bar{v}_{\downarrow}\left(p^{\mu}\right) \gamma^{\mu} \gamma^{5} v_{\uparrow}\left(p^{\mu}\right)=\bar{u}_{\downarrow}\left(p^{\mu}\right) \gamma^{\mu} \gamma^{5} u_{\uparrow}\left(p^{\mu}\right), \\
& \bar{v}_{\downarrow}\left(p^{\mu}\right) \gamma^{\mu} \gamma^{5} v_{\downarrow}\left(p^{\mu}\right)=\bar{u}_{\downarrow}\left(p^{\mu}\right) \gamma^{\mu} \gamma^{5} u_{\downarrow}\left(p^{\mu}\right) .
\end{aligned}
$$

Finally,

$$
\bar{u}_{\uparrow}\left(p^{\mu}\right) \sigma^{\mu \nu} u_{\uparrow}\left(p^{\mu}\right)=\frac{2 i m}{N}\left(\begin{array}{c}
\mathbf{B}^{(+)}(\mathbf{p}, 0) \\
-\mathbf{E}^{(+)}(\mathbf{p}, 0)
\end{array}\right),
$$




$$
\begin{array}{r}
\bar{u}_{\uparrow}\left(p^{\mu}\right) \sigma^{\mu \nu} v_{\uparrow}\left(p^{\mu}\right)=\frac{2 m}{N}\left(\begin{array}{c}
\mathbf{E}^{(+)}(\mathbf{p}, 0) \\
\mathbf{B}^{(+)}(\mathbf{p}, 0)
\end{array}\right), \\
\bar{u}_{\uparrow}\left(p^{\mu}\right) \sigma^{\mu \nu} u_{\downarrow}\left(p^{\mu}\right)=\frac{2 i m \sqrt{2}}{N}\left(\begin{array}{c}
\mathbf{B}^{(+)}(\mathbf{p},-1) \\
\mathbf{E}^{(+)}(\mathbf{p},-1)
\end{array}\right), \\
\bar{u}_{\uparrow}\left(p^{\mu}\right) \sigma^{\mu \nu} v_{\downarrow}\left(p^{\mu}\right)=\frac{2 m \sqrt{2}}{N}\left(\begin{array}{c}
\mathbf{E}^{(+)}(\mathbf{p},-1) \\
\mathbf{B}^{(+)}(\mathbf{p},-1)
\end{array}\right), \\
\bar{u}_{\downarrow}\left(p^{\mu}\right) \sigma^{\mu \nu} u_{\downarrow}\left(p^{\mu}\right)=-\frac{2 i m}{N}\left(\begin{array}{c}
\mathbf{B}^{(+)}(\mathbf{p}, 0) \\
-\mathbf{E}^{(+)}(\mathbf{p}, 0)
\end{array}\right), \\
\bar{u}_{\downarrow}\left(p^{\mu}\right) \sigma^{\mu \nu} v_{\downarrow}\left(p^{\mu}\right)=-\frac{2 m}{N}\left(\begin{array}{c}
\mathbf{E}^{(+)}(\mathbf{p}, 0) \\
\mathbf{B}^{(+)}(\mathbf{p}, 0)
\end{array}\right), \\
\bar{u}_{\downarrow}\left(p^{\mu}\right) \sigma^{\mu \nu} u_{\uparrow}\left(p^{\mu}\right)=-\frac{2 i m \sqrt{2}}{N}\left(\begin{array}{c}
\mathbf{B}^{(+)}(\mathbf{p},+1) \\
-\mathbf{E}^{(+)}(\mathbf{p},+1)
\end{array}\right), \\
\bar{u}_{\downarrow}\left(p^{\mu}\right) \sigma^{\mu \nu} v_{\uparrow}\left(p^{\mu}\right)=-\frac{2 m \sqrt{2}}{N}\left(\begin{array}{c}
\mathbf{E}^{(+)}(\mathbf{p},+1) \\
\mathbf{B}^{(+)}(\mathbf{p},+1)
\end{array}\right) .
\end{array}
$$

All the tensor currents of the type $\bar{v}_{h}\left(p^{\mu}\right) \sigma^{\mu \nu} u_{h^{\prime}}\left(p^{\mu}\right)$ and $\bar{v}_{h}\left(p^{\mu}\right) \sigma^{\mu \nu} v_{h^{\prime}}\left(p^{\mu}\right)$ will differ in the sign from the corresponding expressions (32a $32 \mathrm{~h}$ ) due to $\bar{v}_{h}\left(p^{\mu}\right)=-\bar{u}_{h}\left(p^{\mu}\right) \gamma^{5}$ and the fact that $\left[\gamma^{5}, \sigma^{\mu \nu}\right]_{-}=0$.

\section{APPENDIX B}

Field functions of the $(1 / 2,1 / 2)$ representation (in the momentum space) are deduced in ref. [21] to be:

$$
\begin{gathered}
u^{\mu}(\mathbf{p},+1)=-\frac{N}{m \sqrt{2}}\left(\begin{array}{c}
p_{r} \\
m+\frac{p_{1} p_{r}}{p_{0}+m} \\
i m+\frac{p_{2} p_{r}}{p_{0}+m} \\
\frac{p_{3} p_{r}}{p_{0}+m}
\end{array}\right) \quad, \quad u^{\mu}(\mathbf{p},-1)=\frac{N}{m \sqrt{2}}\left(\begin{array}{c}
p_{l} \\
m+\frac{p_{1} p_{l}}{p_{0}+m} \\
p_{3} \\
-i m+\frac{p_{2} p_{l}}{p_{0}+m} \\
\frac{p_{3} p_{l}}{p_{0}+m}
\end{array}\right), \quad u^{\mu}\left(\mathbf{p}, 0_{t}\right)=\frac{N}{m}\left(\begin{array}{c}
p_{0} \\
p_{1} \\
p_{2} \\
p_{0}+m \\
\frac{p_{2} p_{3}}{p_{0}+m} \\
m+\frac{p_{3}^{2}}{p_{0}+m}
\end{array}\right) .
\end{gathered}
$$


They do not diverge in the massless limit provided that $N=m$ and describe the longitudinal (in the sense $h=0$ ) photons in this limit. If $N=1$ we have "transverse photons" and the divergent behaviour of the gauge parts of these field functions. The negative-energy potentials are obtained by application of the complex conjugation operation (or the $C P$ conjugation, thus obtaining different field operators).

Corresponding strengths are

$$
\begin{aligned}
\mathbf{B}^{(+)}(\mathbf{p},+1) & =-\frac{i N}{2 \sqrt{2} m}\left(\begin{array}{c}
-i p_{3} \\
p_{3} \\
i p_{r}
\end{array}\right)=\mathbf{B}^{(-)}(\mathbf{p},-1) \\
\mathbf{B}^{(+)}(\mathbf{p}, 0) & =\frac{i N}{2 m}\left(\begin{array}{c}
p_{2} \\
-p_{1} \\
0
\end{array}\right)=-\mathbf{B}^{(-)}(\mathbf{p}, 0), \\
\mathbf{B}^{(+)}(\mathbf{p},-1) & =\frac{i N}{2 \sqrt{2} m}\left(\begin{array}{c}
i p_{3} \\
p_{3} \\
-i p_{l}
\end{array}\right)=\mathbf{B}^{(-)}(\mathbf{p},+1)
\end{aligned}
$$

and

$$
\begin{aligned}
\mathbf{E}^{(+)}(\mathbf{p},+1) & =-\frac{i N}{2 \sqrt{2} m}\left(\begin{array}{c}
p_{0}-\frac{p_{1} p_{r}}{p_{0}+m} \\
i p_{0}-\frac{p_{2} p_{r}}{p_{0}+m} \\
-\frac{p_{3} p_{r}}{p_{0}+m}
\end{array}\right)=\mathbf{E}^{(-)}(\mathbf{p},-1) \\
\mathbf{E}^{(+)}(\mathbf{p}, 0) & =\frac{i N}{2 m}\left(\begin{array}{c}
-\frac{p_{1} p_{3}}{p_{0}+m} \\
-\frac{p_{2} p_{3}}{p_{0}+m} \\
p_{0}-\frac{p_{3}^{2}}{p_{0}+m}
\end{array}\right)=-\mathbf{E}^{(-)}(\mathbf{p}, 0), \\
\mathbf{E}^{(+)}(\mathbf{p},-1) & =\frac{i N}{2 \sqrt{2} m}\left(\begin{array}{c}
p_{0}-\frac{p_{1} p_{l}}{p_{0}+m} \\
-i p_{0}-\frac{p_{2} p_{l}}{p_{0}+m} \\
-\frac{p_{3} p_{l}}{p_{0}+m}
\end{array}\right)=\mathbf{E}^{(-)}(\mathbf{p},+1)
\end{aligned}
$$

They were obtained by the application of the formulas: $\mathbf{B}^{( \pm)}(\mathbf{p}, h)= \pm \frac{i}{2 m} \mathbf{p} \times \mathbf{u}^{( \pm)}(\mathbf{p}, h)$ and $\mathbf{E}^{( \pm)}(\mathbf{p}, h)= \pm \frac{i}{2 m} p_{0} \mathbf{u}^{( \pm)}(\mathbf{p}, h) \mp \frac{i}{2 m} \mathbf{p} u^{0( \pm)}(\mathbf{p}, h)$. It is useful to compare these strengths with those presented in [8, p.408] on using the different spinorial basis. Corresponding cross products were also obtained in ref. [21] and it appears that they are related to the 
gauge parts $\left(\sim p^{\mu}\right)$ of the 4-potentials in the momentum space. Relations of cross-products with antisymmetric tensor will be given in a separate paper (preprint EFUAZ FT-99-67 (physics/9907048), Feb. 1999).

[1] L. de Broglie, Compt. Rend. 195 (1932) 862.

[2] P. Jordan, Z. Phys. 93 (1935) 464, ibid. 98 (1936) 759.

[3] R. Kronig, Physica 3 (1936) 1120.

[4] M. H. L. Price Proc. Roy. Soc. A165 (1938) 247.

[5] V. S. Berezinsky, Sov. Phys. JETP 24 (1965) 927.

[6] W. Perkins, Phys. Rev. 137 (1965) B1291; ibid. D5 (1972) 1375.

[7] J. A. McLennan, Phys. Rev. 106 (1957) 821; K. M. Case, Phys. Rev. 107 (1957) 307.

[8] D. V. Ahluwalia and D. J. Ernst, Int. J. Mod. Phys. E2 (1993) 397.

[9] V. V. Dvoeglazov, Speculations in Science and Technology, 21 (1998) 115.

[10] D. V. Ahluwalia, Int. J. Mod. Phys. A11 (1996) 1855.

[11] V. V. Dvoeglazov, Hadronic Journal 20 (1997) 435.

[12] S. Weinberg, The Quantum Theory of Fields, vol. 1.

[13] V. V. Dvoeglazov, Mod. Phys. Lett. A12 (1997) 2741.

[14] V. V. Dvoeglazov, Int. J. Theor. Phys. 34 (1995) 2467.

[15] V. V. Dvoeglazov, Preprint EFUAZ FT-98-56, physics/9801024, Jan. 1998, accepted in Apeiron.

[16] G. Hunter, The $\mathbf{B}^{(3)}$ Field: an Assessment. Preprint, July 1997, to be published.

[17] W. Perkins, email communications (Jan. 1998).

[18] A. O. Barut, Lecture Notes in Physics. Vol. 180 (Berlin-Heidelberg-New York -Tokyo, Springer, 1983), p. 332; A. O. Barut and J. F. Van Huele, Phys. Rev. A32 (1985) 3187; A. O. Barut and G. L. Strobel, Few-Body System 1 (1986) 167

[19] V. V. Dvoeglazov, Fizika B6 (1997) 111.

[20] L. H. Ryder, Quantum Field Theory. (Cambridge University Press, Cambridge, 1985).

[21] V. V. Dvoeglazov, On the Importance of the Normalization. Preprint EFUAZ FT-96-39-REV hepth/9712036), Nov. 1997, accepted in Czech. J. Phys. 